ISBN 978-93-84468-86-6

2016 International Research Conference on Social Sciences, Humanities and Interdisciplinary Studies

(RCSSHIS-2016)

Pattaya (Thailand) Dec. 16-17, 2016

\title{
The Relationship between TOEIC Reading Scores and Achievement Scores in Reading Courses of an English Major Program
}

\author{
Soisithorn Isarankura \\ Dhurakij Pundit University, Bangkok, Thailand
}

\begin{abstract}
This small-scale study seeks to investigate the relationship between scores on the TOEIC Reading section and the achievement scores of four required English reading courses among undergraduate students in an English-major program of a private university in Bangkok. Thailand. The results reveal a significant correlation between the TOEIC reading scores and the achievement test scores of each reading course at the .05 level. The relationship was higher $(r=.695)$ between the TOEIC scores and scores of the first reading course, and it got lower towards the fourth course $(r=.274)$. This could be due to the fact that advanced reading courses are more difficult and they require relatively higher critical thinking skills and the ability to read faster than the more basic courses. Responses from an informal interview with a few students suggest that they lacked the speedreading ability and critical-reading skill. In addition, they stated that they had insufficient strategic testing preparations. Coaching in test-taking techniques and strategies prior to the test seems to be necessary for them. The findings of the study will help the English Major Program planner to find more appropriate directions in designing reading courses that prepare the students to read more quickly and critically and therefore achieve higher scores in the TOEIC reading test.
\end{abstract}

Keywords: Test of English for International Communication, Education Testing Service, TOEIC reading score, achievement test score, English as a foreign language (EFL)

\section{Introduction}

Reading competency is one of the principal foundations for learning and academic achievement. Reading comprehension skill is one among other basic skills employers consider necessary for the workplace. To measure the ability to read English in everyday work situations, many employers have set a certain level of scores on an English proficiency test such as the Test of English for International Communication (TOEIC) as part of the qualifications or requirements for job recruitment and/or promotion within a corporation. As an educational institution, there is a continuous need for the university to work towards producing graduates who meet such requirements of the job market.

The TOEIC test is a well-established English language proficiency test that is used by many corporations and language institutes to measure non-native speakers' everyday English skills that are commonly used in the workplace (Kato, 2005; Kanzaki, 2014). The TOEIC Listening and Reading test has become a widely used English proficiency test in Thailand. Educational Testing Service (ETS), the TOEIC test developer, claims that "TOEIC test scores indicate how well people can communicate in English with others in the global workplace (Educational Testing Service, 2013: 2). The test comprises two sections, one for listening and one for reading, and the test-takers take both sections during the same administration. The listening section contains 100 items to be completed in 45 minutes, and the reading section contains 100 questions to be completed in 75 minutes. The 
scores of the two sections are reported on separate scales. Both scales range from 5 to 495 in increments of 5 . As this paper focuses only on the reading skill, the listening section of the test is not the concern of the present study.

In the university that the author is teaching, the new curriculum of the English-major program requires all fourth-year students to take the TOEIC as partial fulfillment of the students' formative evaluation in one of the major-required courses entitled Career Preparation. This is due to the fact that many leading corporates including airline businesses use the TOEIC as a selection and placement instrument. To enhance the students' reading ability and prepare them for the test, the English-Major Program aims at designing all the reading courses to gear towards developing the students' ability to process large amounts of language quickly and accurately which is an important skill needed to achieve higher TOEIC scores. As an initial step, it is necessary to examine whether there is a relationship between scores of the achievement tests of the four required basic reading courses presently offered in the English program and scores of the TOEIC Reading test taken by 54 English majors.

\section{Review of Literature}

There has been little or no related research conducted with the purpose of examining the relationship between scores on the TOEIC Reading section and the achievement scores of English reading courses obtained by undergraduate students.

Nishizawa, Yoshioka, and Fukada, (2009) investigated the impact of a 4-year extensive reading (ER) program on the TOEIC score among thirty-seven Japanese English as a foreign language (EFL) learners. The results show that the most critical factor for success in an ER program is the amount of reading. A reading amount of 300,000 words was the threshold where many students started to show significant increases in TOEIC scores. Another factor leading to the success of the reading program was to 'start with simple stories' (SSS). This can help the students to avoid concurrent translation from English to their native language and comprehend directly from English texts, which will boost their speed-reading considerably. Nishizawa, Yoshioka, and Fukada suggested that the reading program of 2-3 years should be sufficient for the students to read at least 300,000 words of English texts in order to reach the threshold when the average TOEIC score increases significantly.

Monkolwanich and Wuthisin (2011) examined the correlation between the GPAs of graduates of the hotel and tourism studies department and their TOEIC scores. The correlation was significant at the .05 level with a rather low level of relationship $(\mathrm{r}=.478)$. In this study, the statistical testing result also shows that the graduates' TOEIC scores did not have a significant correlation with the length of time spent prior to getting a job.

\section{Research Question}

Specifically, this study seeks to answer the following question:

- What are the observed correlations between scores of the TOEIC reading section and achievement scores of four major-required reading courses obtained by the fourth-year students in the Englishmajor program?

\section{Research Methodology}

\subsection{Participants}

The participants were 54 fourth-year English majors in a private university, 10 males and 44 females, as shown in Table 1.

TABLE I: Participants

\begin{tabular}{lll}
\hline \hline Gender & Number & $\%$ \\
\hline Male & 10 & 18.52 \\
Female & 44 & 81.48 \\
\hline \hline
\end{tabular}




\subsection{Data Collection Procedure}

All participants took the TOEIC test in the first semester of their fourth year of study. The participants' TOEIC reading test scores were compiled. These scores were compared against the students' final examination scores of the four reading skill courses that the students had taken as major-required subjects in their first and second years of the program. More advanced reading courses are offered in the third year as major-elective courses which are not required for all students to take. The four basic reading skill courses are sequenced as follows:

- EN354 - Reading Skills and Strategies 1

- EN355 - Reading Skills and Strategies 2

- EN356 - Reading Skills and Strategies 3

- EN357 - Critical Reading and Thinking Skills

The final examination scores of the 4 reading courses were collected from the grade and score reports submitted to the English Program by the course instructors at the end of the semester. Due to the fact that the total scores of the four courses varied according to the evaluation plan set forth by each instructor which was not on the same scale, all scores compiled from these four courses were converted to 100 for ease of comparison.

Following the TOEIC test, an informal interview was conducted with a few students to obtain their opinions on the test.

\subsection{Data Analysis}

Descriptive statistics was used to calculate the students' scores into percentage, mean values and standard deviation. Subsequently, Pearson Product-Moment Correlation was conducted to further examine whether there was a correlation between the students' TOEIC scores and achievement scores of the four reading courses.

\section{Results and Discussion}

Table 2 below demonstrates scores of the four English reading skill courses, totaling 400 (100 each $\mathrm{x} 4$ courses), and scores of the TOEIC Reading section, totaling 495. For ease of comparison, the maximum, minimum, and mean scores were calculated into percentages and placed in parentheses.

TABLE II: Achievement Scores of 4 Reading Courses and TOEIC Reading Scores

\begin{tabular}{cccccc}
\hline \hline & Total & Max & Min & Mean & Std. \\
\hline All 4 courses & 400 & $306.6(76.65 \%)$ & $156.7(39.18 \%)$ & $219.21(54.8 \%)$ & 12.4 \\
TOEIC & 495 & $330(66.67 \%)$ & $95(19.19 \%)$ & $183.61(37.09 \%)$ & 60.8 \\
\hline \hline
\end{tabular}

The result in Table 2 shows that the percentage of the mean value of the reading achievement scores (54.8\%) is higher than that of the TOEIC reading score (37.09\%). This finding indicates that the TOEIC reading test is more difficult than the achievement tests of the four courses. This is supported by the minimum TOEIC test score of 95 (19.19\%), which is considerably lower than the minimum achievement test score of $156.7(39.18 \%)$. The higher standard deviation of the TOEIC test as opposed to that of the achievement tests $(60.8<12.4)$ suggests that the TOEIC scores varied substantially among the 54 students.

In order to see whether there is a relationship between the TOEIC scores and achievement scores, Pearson Product-Moment Correlation was conducted. The result shows a medium-level relationship $(\mathrm{r}=.565)$; the correlation is statistically significant $(* \mathrm{p}<.05)$, as shown in Table 3.

TABLE III: Correlations between Scores of 4 Reading Courses and TOEIC Reading Scores

\begin{tabular}{lll}
\hline \hline & \multicolumn{2}{l}{ TOEIC } \\
\cline { 2 - 3 } & $\mathrm{r}$ & Sig. \\
\hline 4 Courses & $.565 *$ & .000 \\
\hline \multicolumn{3}{c}{$* \mathrm{p}<.05$}
\end{tabular}

When examining the achievement scores of each reading course as presented in Table 4, one can see that the mean scores for the three basic courses, Reading Skills and Strategies $1-3$ increased slightly (i.e. 54.50, 57.53, 60.19 , respectively) as the students' reading proficiency developed. However, the mean score of the fourth 
course, EN357 - Critical Reading and Thinking Skills, dropped to 46.99 out of the total score of 100. This is possibly due to the fact that many students had difficulty utilizing critical thinking skills while reading.

TABLE IV Achievement Scores of Each Reading Course and TOEIC Reading Scores

\begin{tabular}{|c|c|c|c|c|c|}
\hline \multicolumn{6}{|c|}{ Students' Achievement Scores $(\mathrm{n}=54)$} \\
\hline & Total & $\operatorname{Max}$ & Min & Mean & Std. \\
\hline EN354 & 100 & 83.3 & 26.7 & 54.50 & 4.13 \\
\hline EN355 & 100 & 83.3 & 35 & 57.53 & 3.09 \\
\hline EN356 & 100 & 100 & 26.7 & 60.19 & 4.46 \\
\hline EN357 & 100 & 72.5 & 22.5 & 46.99 & 5.27 \\
\hline \multicolumn{6}{|c|}{ TOEIC Reading Scores $(n=54)$} \\
\hline & Total & Max & Min & Mean & Std. \\
\hline TOEIC & 495 & $330(66.67 \%)$ & $95(19.19 \%)$ & $183.61(37.09 \%)$ & 60.8 \\
\hline
\end{tabular}

To determine whether a correlation exists between the TOEIC reading test and the achievement scores of each reading course, Pearson Product-Moment Correlation was used to analyze the data. The results reveal that there is a relationship between the TOEIC scores and the scores of each reading course. The correlation is significant at the .05 level, as displayed in Table 5. One can see that the scores obtained from EN354 - Reading Skills and Strategies 1 and the TOEIC reading scores show a high level of relationship $(\mathrm{r}=.695, \mathrm{p}<.05)$, while the scores of EN355 and EN356 Reading Skills and Strategies 2 and 3 had a medium level of relationship with the TOEIC scores $(r=.430$, and $r=.300$, respectively). Of the four required reading courses, EN357 Critical Reading and Thinking Skills had the lowest level of relationship with the TOEIC scores $(r=.274, p<.05)$.

TABLE V: Correlations between Scores of Each Reading Course and TOEIC Reading Scores

\begin{tabular}{lll}
\hline \hline & TOEIC & \\
\cline { 2 - 3 } & $\mathrm{r}$ & Sig. \\
\hline EN354 & $.695^{*}$ & .000 \\
\hline EN355 & $.430^{*}$ & .001 \\
\hline EN356 & $.300^{*}$ & .027 \\
\hline EN357 & $.274^{*}$ & .045 \\
\hline \multicolumn{3}{c}{$* \mathrm{p}<.05$}
\end{tabular}

An informal post-exam interview was conducted with a few students following the TOEIC test, the following problems can be summarized.

1. The students complained that the TOEIC reading test was too long for them to finish within the allocated time.

One female test-taker said, "I believe that most students find reading is the most difficult skill. We need to cope with vocabulary and comprehending the texts under time constraints. The TOEIC reading test is very long; we have to read very quickly, otherwise we cannot finish the test within the allocated time".

2. The students felt that they lacked time management skill. They spent too much time reading short passages in the earlier sections and thus had no time to finish reading longer passages in the latter half of the test.

According to one male student, "my TOEIC reading score was low because I spent too much time trying to comprehend each passage and ended up in being unable to finish the test in time. I think I need to learn the strategies to do the test".

3. The students had limited knowledge of vocabulary.

In view of this problem, a female student responded that she found a number of unfamiliar words in several passages. She said, "Most passages are related to business context and many words are supposed to be used in the workplace". "I think that texts concerning business should be introduced in more advanced reading courses so that the students can familiarize themselves with the vocabulary necessary for the future career". 
4. In order to understand the meaning of the text, many students are accustomed to translating from English into Thai. This method tends to be time-consuming and does not work very well when speed-reading is required.

"I am in the habit of translating the sentences into Thai in order to understand the meaning of the text. This makes me read English slowly," said one male student.

\section{Results and Implications}

From the research findings, the discussions and implications are presented based on the research question as follows.

Due to the low maximum, minimum and mean scores obtained from the TOEIC reading test, one can imagine that the level of difficulty of the TOEIC test is higher than the achievement test of each required reading course in the English major program. Such difficulty possibly arises from the following factors.

1. Insufficient knowledge of vocabulary

2. Complex sentence structures

3. Lack of the critical reading skill

4. Lack of the speed reading skill

5. Lack of strategic testing preparations including the time management skill

Therefore, in order to develop the students' reading skills needed to achieve higher TOEIC scores, the English Major program should provide students with the coaching in test-taking techniques and strategies, which may be included in one or more reading courses in the program. The TOEIC test requires test-takers to have the ability to read quickly in a short time. This speed reading skill has to be taught along with the techniques to effectively utilize the allocated test time so that the students can finish the reading section comfortably within 75 minutes.

\section{Acknowledgements}

I wish to thank the participants for their willingness to offer all requested information. My profound appreciation goes to Assistant Professor Dr. Thuntuch Viphatphumiprathes for his assistance in analysing the data. I also owe a special word of thanks to Mr. Christopher Dean Moszuti, who gave valuable suggestions and edited this paper.

\section{References}

[1] Educational Testing Service. (2013). TOEIC user guide-listening and reading. Princeton, NJ: Educational Testing Service. Available: http://www.ets.org/Media/Tests/Test_of_English_for_International_Communication/TOEIC_User _Gd.pdf

[2] M. Kanzaki, "TOEIC Survey: speaking vs. listening and reading," in Proc. JALT 2014 Conference Proceedings, pp 626-636.

[3] S. Kato, How language learning strategies affect English proficiency in Japanese university students, Journal of the Faculty of Human Studies Bunkyo Gakuin University, 7 (1), 2005, pp 239-262.

[4] C. Monkolwanich \& M. Wuthisin, The correlation between getting a job and the TOEIC score as well as the relation between getting a job and their cumulative GPA, Journal of Cultural Approach, Vol. 13 (23), 2011, pp 18-26.

[5] H. Nishizawa, T. Yoshioka \& M. Fukada, "The impact of a 4-year extensive reading program," in Proc. JALT 2009 Conference Proceedings, 2009, pp 632-640. 